\title{
Intersecting and cross-intersecting families of labeled sets
}

\author{
Peter Borg \\ Department of Mathematics, Junior College, University of Malta \\ Pjazza Guzè Debono, Msida MSD 1252, Malta \\ p.borg.02@cantab.net
}

Submitted: Jan 6, 2008; Accepted: Mar 17, 2008; Published: Mar 27, 2008

Mathematics Subject Classification: 05D05

\begin{abstract}
A family $\mathcal{A}$ of sets is said to be intersecting if any two sets in $\mathcal{A}$ intersect. Families $\mathcal{A}_{1}, \ldots, \mathcal{A}_{p}$ are said to be cross-intersecting if, for any $i, j \in\{1, \ldots, p\}$ such that $i \neq j$, any set in $\mathcal{A}_{i}$ intersects any set in $\mathcal{A}_{j}$.

For $\mathbf{k}=\left(k_{1}, \ldots, k_{n}\right) \in \mathbb{N}^{n}, 2 \leq k_{1} \leq \ldots \leq k_{n}$, let $\mathcal{L}_{\mathbf{k}}$ be the family of labeled $n$-sets given by $\mathcal{L}_{\mathbf{k}}:=\left\{\left\{\left(1, l_{1}\right), \ldots,\left(n, l_{n}\right)\right\}: l_{i} \in\left\{1, \ldots, k_{i}\right\}, i=1, \ldots, n\right\}$. We point out a relationship between intersecting families and cross-intersecting families of labeled sets, and we show that, if $\mathcal{A}_{1}, \ldots, \mathcal{A}_{p}$ are cross-intersecting sub-families of $\mathcal{L}_{\mathbf{k}}$, then

$$
\sum_{j=1}^{p}\left|\mathcal{A}_{j}\right| \leq \begin{cases}k_{1} k_{2} \ldots k_{n} & \text { if } p \leq k_{1} \\ p k_{2} \ldots k_{n} & \text { if } p \geq k_{1}\end{cases}
$$

We also determine the cases of equality. We then obtain a more general inequality, a special case of which is a sharp bound for cross-intersecting families of permutations.
\end{abstract}

\section{Old and new intersection results for labeled sets}

We start with some standard notation for sets. $\mathbb{N}$ is the set of positive integers $\{1,2, \ldots\}$. For $m, n \in \mathbb{N}, m<n$, the set $\{m, m+1, \ldots, n\}$ is denoted by $[m, n]$, and if $m=1$ then we also write $[n]$. The power set $\{A: A \subseteq X\}$ of a set $X$ is denoted by $2^{X}$, and a uniform sub-family $\{Y \subseteq X:|Y|=r\}$ of $2^{X}$ is denoted by $\left(\begin{array}{l}X \\ r\end{array}\right)$.

We denote the union of all sets in a family $\mathcal{F}$ by $U(\mathcal{F})$. For $u \in U(\mathcal{F})$, the family of sets in $\mathcal{F}$ that contain $u$ is called a star of $\mathcal{F}$ with centre $u$.

A family $\mathcal{A}$ is said to be intersecting if any two sets in $\mathcal{A}$ intersect. Note that a star of a family is trivially intersecting.

The classical Erdôs-Ko-Rado (EKR) Theorem [6] says that, for $r \leq n / 2$, an intersecting sub-family $\mathcal{A}$ of $\left(\begin{array}{c}{[n]} \\ r\end{array}\right)$ has size at most $\left(\begin{array}{c}n-1 \\ r-1\end{array}\right)$, i.e. the size of a star of $\left(\begin{array}{c}{[n]} \\ r\end{array}\right)$. By the 
Hilton-Milner Theorem [9], if $r<n / 2$ then $\mathcal{A}$ attains the bound if and only if $\mathcal{A}$ is a star of $\left(\begin{array}{c}{[n]} \\ r\end{array}\right)$. Many results were inspired by the EKR Theorem; see [5].

Families $\mathcal{A}_{1}, \ldots, \mathcal{A}_{p}$ are said to be cross-intersecting if, for any $i, j \in[p]$ such that $i \neq j$, any set in $\mathcal{A}_{i}$ intersects any set in $\mathcal{A}_{j}$.

Hilton [8] determined the following nice EKR-type result for cross-intersecting subfamilies of $\left(\begin{array}{c}{[n]} \\ r\end{array}\right)(r \leq n / 2)$.

Theorem 1.1 (Hilton [8]) Let $r \leq n / 2$ and $p \geq 2$. Let $\mathcal{A}_{1}, \ldots, \mathcal{A}_{p}$ be cross-intersecting sub-families of $\left(\begin{array}{c}{[n]} \\ r\end{array}\right)$. Then

$$
\sum_{j=1}^{p}\left|\mathcal{A}_{j}\right| \leq \begin{cases}\left(\begin{array}{l}
n \\
r
\end{array}\right) & \text { if } p \leq \frac{n}{r} \\
p\left(\begin{array}{l}
n-1 \\
r-1
\end{array}\right) & \text { if } p \geq \frac{n}{r}\end{cases}
$$

Unless $p=2=n / r$, the bound is attained if and only if one of the following holds:

(i) $p<n / r$ and, for some $q \in[p], \mathcal{A}_{q}=\left(\begin{array}{c}{[n]} \\ r\end{array}\right)$ and $\mathcal{A}_{j}=\emptyset$ for all $j \in[p] \backslash\{q\}$;

(ii) $p>n / r$ and $\left|\mathcal{A}_{1}\right|=\ldots=\left|\mathcal{A}_{p}\right|=\left(\begin{array}{c}n-1 \\ r-1\end{array}\right)$;

(iii) $p=n / r$ and $\mathcal{A}_{1}, \ldots, \mathcal{A}_{p}$ are as in (i) or (ii).

The EKR Theorem follows from this result: set $p>n / r$ and $\mathcal{A}_{1}=\ldots=\mathcal{A}_{p}$. In [2] it is shown that in case (ii) we must have $\mathcal{A}_{1}=\ldots=\mathcal{A}_{p}=\left\{A \in\left(\begin{array}{c}{[n]} \\ r\end{array}\right): i \in A\right\}$ for some $i \in[n]$.

For $\mathbf{k}=\left(k_{1}, \ldots, k_{n}\right), k_{1}, \ldots, k_{n} \in \mathbb{N} \backslash\{1\}$, we define the family $\mathcal{L}_{\mathbf{k}}$ of labeled $n$-sets by

$$
\mathcal{L}_{\mathbf{k}}:=\left\{\left\{\left(1, l_{1}\right), \ldots,\left(n, l_{n}\right)\right\}: l_{i} \in\left[k_{i}\right], i=1, \ldots, n\right\} .
$$

An equivalent formulation for $\mathcal{L}_{\mathbf{k}}$ is $\left[k_{1}\right] \times\left[k_{2}\right] \times \ldots \times\left[k_{n}\right]$, but it is more convenient to work with $n$-sets than work with $n$-tuples (the alternative formulation demands that we change the setting of families of sets to one of sets of $n$-tuples).

In this note we are concerned with the sizes of intersecting and cross-intersecting families of labeled $n$-sets. Note that, if we allow $k_{i}=1$ for some $i \in[n]$, then the problem becomes trivial because we get that all sets contain the point $(i, 1)$.

The obvious EKR-type problem for labeled sets was treated by Berge [1].

Theorem 1.2 (Berge [1]) Let $m \in[n]$ such that $k_{m}=\min \left\{k_{i}: i \in[n]\right\}$. If $\mathcal{A}$ is an intersecting sub-family of $\mathcal{L}_{\mathbf{k}}$, then the size of $\mathcal{A}$ is at most $\left|\mathcal{L}_{\mathbf{k}}\right| / k_{m}=k_{1} k_{2} \ldots k_{n} / k_{m}$, i.e. the size of a star of $\mathcal{L}_{\mathbf{k}}$ with centre $(m, 1)$.

We shall reproduce the remarkably short proof of this result.

For an integer $q$, let $\theta_{\mathbf{k}}^{q}: \mathcal{L}_{\mathbf{k}} \rightarrow \mathcal{L}_{\mathbf{k}}$ be the translation operation defined by

$$
\theta_{\mathbf{k}}^{q}(A):=\left\{\left(a, b+q \bmod k_{a}\right):(a, b) \in A\right\},
$$

and define $\Theta_{\mathrm{k}}^{q}: 2^{\mathcal{L}_{\mathbf{k}}} \rightarrow 2^{\mathcal{L}_{\mathbf{k}}}$ by

$$
\Theta_{\mathbf{k}}^{q}(\mathcal{F}):=\left\{\theta_{\mathbf{k}}^{q}(A): A \in \mathcal{F}\right\}
$$


Now let $m$ and $\mathcal{A}$ be as in Theorem 1.2. For any $A \in \mathcal{A}$ and $q \in\left[k_{m}-1\right]$, we have $\theta_{\mathbf{k}}^{q}(A) \cap A=\emptyset$ and hence $\theta_{\mathbf{k}}^{q}(A) \notin \mathcal{A}$. Therefore $\mathcal{A}, \Theta_{\mathbf{k}}^{1}(\mathcal{A}), \ldots, \Theta_{\mathbf{k}}^{k_{m}-1}(\mathcal{A})$ are $k_{m}$ disjoint sub-families of $\mathcal{L}_{\mathbf{k}}$. So $k_{m}|\mathcal{A}| \leq\left|\mathcal{L}_{\mathbf{k}}\right|=k_{1} k_{2} \ldots k_{n}$ and hence Theorem 1.2.

Livingston [10] determined which families $\mathcal{A}$ attain the bound in Theorem 1.2 for the case when the $k_{i}$ 's are all the same.

Theorem 1.3 (Livingston [10]) If $3 \leq k_{1}=k_{2}=\ldots=k_{n}$ and $\mathcal{A}$ is a largest intersecting sub-family of $\mathcal{L}_{\mathbf{k}}$, then $\mathcal{A}$ is a star of $\mathcal{L}_{\mathbf{k}}$.

Using the shifting technique (see [7]) in an inductive argument, we can extend Theorem 1.3 to the following result.

Theorem 1.4 Let $m \in[n]$ such that $k_{m}=\min \left\{k_{i}: i \in[n]\right\}$. Suppose $k_{m} \geq 3$ and $\mathcal{A}$ is a largest intersecting sub-family of $\mathcal{L}_{\mathrm{k}}$. Then $\mathcal{A}$ is a star of $\mathcal{L}_{\mathrm{k}}$ with centre in $C:=\left\{(i, l): i \in[n], k_{i}=k_{m}, l \in\left[k_{m}\right]\right\}$.

Proof. We use induction on $\sum_{i=1}^{n} k_{i}$. The case $k_{i}=k_{m}$ for $i=1, \ldots, n$ is Theorem 1.3, so we assume there exists $h \in[n]$ such that $k_{h}>k_{m}$. We may assume $m=1$ and $h=n$. Let $\delta: \mathcal{A} \rightarrow \mathcal{L}_{\mathrm{k}}$ be the shift operation defined by

$$
\delta(A):= \begin{cases}\left(A \backslash\left\{\left(n, k_{n}\right)\right\}\right) \cup\{(n, 1)\} & \text { if }\left(n, k_{n}\right) \in A ; \\ A & \text { otherwise }\end{cases}
$$

and let $\mathcal{B}:=\{\delta(A): A \in \mathcal{A}, \delta(A) \notin \mathcal{A}\} \cup\{A \in \mathcal{A}: \delta(A) \in \mathcal{A}\}$. Clearly $|\mathcal{B}|=|\mathcal{A}|$.

We now show that, since $\mathcal{A}$ is intersecting, $\mathcal{B}$ is intersecting and, moreover, no two sets in $\mathcal{B}$ intersect only on $\left(n, k_{n}\right)$. Let $B_{1}$ and $B_{2}$ be two arbitrary sets in $\mathcal{B}$. If neither set contains $\left(n, k_{n}\right)$ then either they intersect on $(n, 1)$ or at least one of them does not contain $(n, 1)$ either and hence must intersect the other. Now suppose without loss of generality $\left(n, k_{n}\right) \in B_{1}$. Then $B_{1}, \delta\left(B_{1}\right) \in \mathcal{A}$. If $\left(n, k_{n}\right) \in B_{2}$ then $B_{2}$ is also in $\mathcal{A}$ and hence $\emptyset \neq \delta\left(B_{1}\right) \cap B_{2}=\left(B_{1} \cap B_{2}\right) \backslash\left\{\left(n, k_{n}\right)\right\}$. Suppose $\left(n, k_{n}\right) \notin B_{2}$. If $B_{2} \in \mathcal{A}$ then $\left(n, k_{n}\right) \notin$ $B_{1} \cap B_{2} \neq \emptyset$ is obvious. If $B_{2} \notin \mathcal{A}$ then $(n, 1) \in B_{2}$, the set $A_{2}:=\left(B_{2} \backslash\{(n, 1)\}\right) \cup\left\{\left(n, k_{n}\right)\right\}$ is in $\mathcal{A}$, and hence we have $\left(n, k_{n}\right) \notin B_{1} \cap B_{2}=\delta\left(B_{1}\right) \cap A_{2} \neq \emptyset$.

Defining $\mathcal{B}_{1}:=\left\{B \in \mathcal{B}:\left(n, k_{n}\right) \notin B\right\}, \mathcal{B}_{2}:=\left\{B \in \mathcal{B}:\left(n, k_{n}\right) \in B\right\}$ and $\mathcal{B}_{2}^{\prime}:=$ $\left\{B \backslash\left\{\left(n, k_{n}\right)\right\}: B \in \mathcal{B}_{2}\right\}$, it follows that $\mathcal{B}_{1} \cup \mathcal{B}_{2}^{\prime}$ is intersecting. Now $\mathcal{B}_{1} \subset \mathcal{L}_{\mathbf{k}_{1}}$ and $\mathcal{B}_{2}^{\prime} \subset \mathcal{L}_{\mathbf{k}_{2}}$, where $\mathbf{k}_{1}=\left(k_{1}, \ldots, k_{n-1}, k_{n}-1\right)$ and $\mathbf{k}_{2}=\left(k_{1}, \ldots, k_{n-1}\right)$. Let $\mathcal{S}:=\{S \in$ $\left.\mathcal{L}_{\mathrm{k}}:(1,1) \in S\right\}$, and define $\mathcal{S}_{1}, \mathcal{S}_{2}$ and $\mathcal{S}_{2}^{\prime}$ similarly to $\mathcal{B}_{1}, \mathcal{B}_{2}$ and $\mathcal{B}_{2}^{\prime}$ respectively. By the inductive hypothesis, $\left|\mathcal{B}_{1}\right| \leq\left|\mathcal{S}_{1}\right|$ and $\left|\mathcal{B}_{2}^{\prime}\right| \leq\left|\mathcal{S}_{2}^{\prime}\right|$. So $|\mathcal{A}| \leq|\mathcal{S}|$ as $|\mathcal{A}|=|\mathcal{B}|=\left|\mathcal{B}_{1}\right|+\left|\mathcal{B}_{2}^{\prime}\right|$ and $|\mathcal{S}|=\left|\mathcal{S}_{1}\right|+\left|\mathcal{S}_{2}^{\prime}\right|$. Since $\mathcal{A}$ is a largest intersecting sub-family of $\mathcal{L}_{\mathrm{k}}$, we actually have $|\mathcal{A}|=|\mathcal{S}|$, which implies $\left|\mathcal{B}_{1}\right|=\left|\mathcal{S}_{1}\right|$ and $\left|\mathcal{B}_{2}^{\prime}\right|=\left|\mathcal{S}_{2}^{\prime}\right|$. By noting that $C \subseteq U\left(\mathcal{L}_{\mathbf{k}_{2}}\right)$ (since $\left.k_{n}>k_{1}\right)$ and applying the inductive hypothesis, we get $\mathcal{B}_{2}^{\prime}=\left\{B \in \mathcal{L}_{\mathbf{k}_{2}}:(a, b) \in B\right\}$ for some $(a, b) \in C$. Thus, for any set in $\left\{A \in \mathcal{L}_{\mathbf{k}_{1}}:(a, b) \notin A\right\}$, there clearly exists a set in $\mathcal{B}_{2}^{\prime}$ that does not intersect with it. Since $\mathcal{B}_{1} \cup \mathcal{B}_{2}^{\prime}$ is intersecting, it follows that all sets in $\mathcal{B}_{1} \cup \mathcal{B}_{2}^{\prime}$ contain $(a, b)$. So $\mathcal{B} \subseteq \mathcal{C}:=\left\{A \in \mathcal{L}_{\mathrm{k}}:(a, b) \in A\right\}$. Since $(a, b) \notin\left\{(n, 1),\left(n, k_{n}\right)\right\}$, we deduce that $\mathcal{A} \subseteq \mathcal{C}$. By maximality of $\mathcal{A}, \mathcal{A}=\mathcal{C}$. Hence result. 
We will now use Theorems 1.2 and 1.4 to obtain the following 'labeled sets' analogue of Theorem 1.1.

Theorem 1.5 Let $m \in[n]$ such that $k_{m}=\min \left\{k_{i}: i \in[n]\right\}$, and let $C:=\{(i, l): i \in$ $\left.[n], k_{i}=k_{m}, l \in\left[k_{m}\right]\right\}$. Let $\mathcal{A}_{1}, \ldots, \mathcal{A}_{p}$ be cross-intersecting sub-families of $\mathcal{L}_{\mathbf{k}}$. Then

$$
\sum_{j=1}^{p}\left|\mathcal{A}_{j}\right| \leq \begin{cases}\left|\mathcal{L}_{\mathbf{k}}\right|=k_{1} k_{2} \ldots k_{n} & \text { if } p \leq k_{m} \\ p\left|\left\{A \in \mathcal{L}_{\mathbf{k}}:(m, 1) \in A\right\}\right|=p \frac{k_{1} k_{2} \ldots k_{n}}{k_{m}} & \text { if } p \geq k_{m}\end{cases}
$$

If $k_{m} \geq 3$, then the bound is attained if and only if one of the following holds:

(i) $p<k_{m}$ and, for some $q \in[p], \mathcal{A}_{q}=\mathcal{L}_{\mathbf{k}}$ and $\mathcal{A}_{j}=\emptyset$ for all $j \in[p] \backslash\{q\}$;

(ii) $p>k_{m}$ and $\mathcal{A}_{1}=\ldots=\mathcal{A}_{p}=\left\{A \in \mathcal{L}_{\mathrm{k}}:(a, b) \in A\right\}$ for some $(a, b) \in C$;

(iii) $p=k_{m}$ and $\mathcal{A}_{1}, \ldots, \mathcal{A}_{p}$ are as in (i) or (ii).

Proof. Let $k_{n+1}:=p$ and $\mathbf{k}^{\prime}:=\left(k_{1}, \ldots, k_{n}, k_{n+1}\right)$. For each $j \in\left[k_{n+1}\right]$, let

$$
\mathcal{A}_{j}^{\prime}:=\left\{A \cup\{(n+1, j)\}: A \in \mathcal{A}_{j}\right\} \text {. }
$$

Now let $\mathcal{A}$ be the the disjoint union $\mathcal{A}_{1}^{\prime} \cup \mathcal{A}_{2}^{\prime} \cup \ldots \cup \mathcal{A}_{k_{n+1}}^{\prime}$, which is a sub-family of $\mathcal{L}_{\mathrm{k}^{\prime}}$. Take two arbitrary sets $A_{1}$ and $A_{2}$ in $\mathcal{A}$. So $A_{1} \in \mathcal{A}_{j_{1}}^{\prime}$ and $A_{2} \in \mathcal{A}_{j_{2}}^{\prime}$ for some $j_{1}, j_{2} \in\left[k_{n+1}\right]$. Also $B_{1}:=A_{1} \backslash\left\{\left(n+1, j_{1}\right)\right\}$ is in $\mathcal{A}_{j_{1}}$ and $B_{2}:=A_{2} \backslash\left\{\left(n+1, j_{2}\right)\right\}$ is in $\mathcal{A}_{j_{2}}$. If $j_{1}=j_{2}$ then $A_{1}$ and $A_{2}$ intersect on $\left(n+1, j_{1}\right)$. If $j_{1} \neq j_{2}$ then $\mathcal{A}_{j_{1}}$ and $\mathcal{A}_{j_{2}}$ are cross-intersecting, and this gives us $B_{1} \cap B_{2} \neq \emptyset$ and hence $A_{1} \cap A_{2} \neq \emptyset$. So $\mathcal{A}$ is an intersecting sub-family of $\mathcal{L}_{\mathbf{k}^{\prime}}$. Since $|\mathcal{A}|=\sum_{j=1}^{k_{n+1}}\left|\mathcal{A}_{j}\right|$, it follows by Theorem 1.2 (with $\mathbf{k}^{\prime}$ instead of $\mathbf{k}$ ) that

$$
\sum_{j=1}^{k_{n+1}}\left|\mathcal{A}_{j}\right| \leq \frac{\left|\mathcal{L}_{\mathbf{k}^{\prime}}\right|}{\min \left\{k_{i}: i \in[n+1]\right\}}=\frac{k_{1} k_{2} \ldots k_{n+1}}{\min \left\{k_{m}, k_{n+1}\right\}},
$$

which immediately yields the desired upper bound.

Now suppose $k_{m} \geq 3$. It is straightforward that the upper bound is attained in each of the cases (i), (ii) and (iii). We now assume that the upper bound is attained and prove the converse. We obviously divide the problem into the following cases.

Case 1: $k_{n+1}<k_{m}$. So we have $|\mathcal{A}|=\frac{\left|\mathcal{L}_{\mathrm{k}^{\prime}}\right|}{k_{n+1}}$.

Suppose first that $k_{n+1} \geq 3$. Since $k_{n+1}<k_{m} \leq k_{i}$ for all $i \in[n]$, it follows by Theorem 1.4 that $\mathcal{A}=\left\{A \in \mathcal{L}_{\mathbf{k}^{\prime}}:(n+1, q) \in A\right\}$ for some $q \in\left[k_{n+1}\right]$. By construction of $\mathcal{A}$, we get $\mathcal{A}_{q}=\mathcal{L}_{\mathrm{k}}$ and $\mathcal{A}_{j}=\emptyset$ for all $j \in\left[k_{n+1}\right] \backslash\{q\}$.

Now suppose $k_{n+1}=2$. Let $k_{n+1}^{\prime}:=k_{m}$, and let $\mathbf{k}^{\prime \prime}:=\left(k_{1}, \ldots, k_{n}, k_{n+1}^{\prime}\right)$. Since $k_{n+1}^{\prime} \geq 3$ and $|\mathcal{A}|=\frac{\left|\mathcal{L}_{\mathbf{k}^{\prime}}\right|}{k_{n+1}}=\frac{\left|\mathcal{L}_{\mathbf{k}^{\prime \prime}}\right|}{k_{n+1}^{\prime}}$, Theorem 1.2 tells us that $\mathcal{A}$ is a largest intersecting sub-family of $\mathcal{L}_{\mathbf{k}^{\prime \prime}}$. Thus, by Theorem $1.4, \mathcal{A}=\left\{A \in \mathcal{L}_{\mathbf{k}^{\prime \prime}}:(a, b) \in A\right\}$ for some $(a, b) \in U\left(\mathcal{L}_{\mathbf{k}^{\prime \prime}}\right)$. Since $\mathcal{A} \subseteq \mathcal{L}_{\mathbf{k}^{\prime}}$, we actually have $(a, b) \in U\left(\mathcal{L}_{\mathbf{k}^{\prime}}\right)$. Suppose $a \neq n+1$; then $|\mathcal{A}|=\frac{\left|\mathcal{L}_{\mathbf{k}^{\prime}}\right|}{k_{a}}$ and hence, since $|\mathcal{A}|=\frac{\left|\mathcal{L}_{\mathrm{k}^{\prime}}\right|}{k_{n+1}}$, we get $k_{a}=k_{n+1}$, which contradicts $k_{a} \geq k_{n+1}^{\prime} \geq 3>k_{n+1}$. So $a=n+1$. By construction of $\mathcal{A}$, we get $\mathcal{A}_{b}=\mathcal{L}_{\mathrm{k}}$ and $\mathcal{A}_{j}=\emptyset$ for all $j \in\left[k_{n+1}\right] \backslash\{b\}$. 
Case 2: $k_{n+1}>k_{m}$. So $k_{m}=\min \left\{k_{1}, \ldots, k_{n}, k_{n+1}\right\}$. By Theorem 1.4, we must have $\mathcal{A}=\left\{A \in \mathcal{L}_{\mathbf{k}^{\prime}}:(a, b) \in A\right\}$ for some $(a, b) \in D:=\left\{(i, l): i \in[n+1], k_{i}=k_{m}, l \in\left[k_{m}\right]\right\}$. Since $k_{n+1}>k_{m}, a \neq n+1$ and $D=C$. Thus, by construction of $\mathcal{A}$, for each $j \in\left[k_{n+1}\right]$ we have $\mathcal{A}_{j}^{\prime}=\left\{A \cup\{(n+1, j)\}:(a, b) \in A \in \mathcal{L}_{\mathrm{k}}\right\}$ and hence $\mathcal{A}_{j}=\left\{A \in \mathcal{L}_{\mathrm{k}}:(a, b) \in A\right\}$.

Case 3: $k_{n+1}=k_{m}$. By Theorem 1.4, $\mathcal{A}=\left\{A \in \mathcal{L}_{\mathbf{k}^{\prime}}:(a, b) \in A\right\}$ for some $(a, b) \in$ $C \cup\left(\{n+1\} \times\left[k_{n+1}\right]\right)$. Therefore, if $(a, b) \in\{n+1\} \times\left[k_{n+1}\right]$ then $\mathcal{A}_{1}, \ldots, \mathcal{A}_{k_{n+1}}$ are as in Case 1 , and if $(a, b) \in C$ then $\mathcal{A}_{1}, \ldots, \mathcal{A}_{k_{n+1}}$ are as in Case 2.

\section{Translation invariant families of labeled sets}

In this section, we generalize the sharp bounds in the above results.

Definition 2.1 We say that $\mathcal{F}$ is translation invariant with respect to $\mathcal{L}_{\mathbf{k}}$ if $\mathcal{F} \subseteq \mathcal{L}_{\mathbf{k}}$ and $\Theta_{\mathbf{k}}^{1}(\mathcal{F})=\mathcal{F}$.

When it is clear from the context that we are considering $\mathcal{F} \subseteq \mathcal{L}_{\mathbf{k}}$, then, if $\Theta_{\mathbf{k}}^{1}(\mathcal{F})=\mathcal{F}$, we simply say that $\mathcal{F}$ is translation invariant.

For any $r, s \in \mathbb{N}$ with $r \leq s$, let

$$
\mathcal{P}_{r, s}:=\left\{\left\{\left(1, l_{1}\right), \ldots,\left(r, l_{r}\right)\right\}: l_{1}, \ldots, l_{r} \text { are distinct elements of }[s]\right\} .
$$

$\mathcal{P}_{r, s}$ is an example of a translation invariant sub-family of $\mathcal{L}_{\mathbf{k}}$ with the $n=r$ entries of $\mathbf{k}$ being all $s$. The special family $\mathcal{P}_{n, n}$ describes permutations of $[n]$.

One can find various other examples of translation invariant families. For example, if $k_{1}=\ldots=k_{m} \leq k_{m+1}=\ldots=k_{n}$, then clearly $\left\{\left\{\left(1, l_{1}\right), \ldots,\left(m, l_{1}\right),\left(m+1, l_{2}\right), \ldots,\left(n, l_{2}\right)\right\}\right.$ : $\left.l_{1} \in\left[k_{1}\right], l_{2} \in\left[k_{m+1}\right]\right\}$ is a translation invariant sub-family of $\mathcal{L}_{\mathbf{k}}$.

The following is a straightforward result.

Proposition 2.2 Let $\mathcal{F} \subseteq \mathcal{L}_{\mathbf{k}}$. $\mathcal{F}$ is translation invariant if and only if $\Theta_{\mathbf{k}}^{q}(\mathcal{F})=\mathcal{F}$ for any $q \in \mathbb{N}$.

Theorem 2.3 Let $\mathcal{F}$ be translation invariant with respect to $\mathcal{L}_{\mathbf{k}}$. Let $m \in[n]$ such that $k_{m}=\min \left\{k_{i}: i \in[n]\right\}$. Then the size of an intersecting sub-family of $\mathcal{F}$ is at most $|\mathcal{F}| / k_{m}$, which is the size of a star of $\mathcal{F}$ with centre $(m, 1)$.

Proof. For each $j \in\left[k_{m}\right]$, let $\mathcal{F}_{j}:=\{F \in \mathcal{F}:(m, j) \in F\}$ (i.e. the star of $\mathcal{F}$ with centre $(m, j))$. So the families $\mathcal{F}_{1}, \ldots, \mathcal{F}_{k_{m}}$ partition $\mathcal{F}$. Clearly, for any $q \in \mathbb{N}$, $\theta_{\mathbf{k}}^{q}$ is an injective function. Consider $j_{1}, j_{2} \in\left[k_{m}\right], j_{1}<j_{2}$. $\theta_{\mathbf{k}}^{j_{2}-j_{1}}$ maps any set $F_{j_{1}} \in \mathcal{F}_{j_{1}}$ to a set $F_{j_{2}} \in\left\{A \in \mathcal{L}_{\mathbf{k}}:\left(m, j_{2}\right) \in A\right\}$, and $F_{j_{2}} \in \mathcal{F}_{j_{2}}$ by Proposition 2.2 ; so $\left|\mathcal{F}_{j_{1}}\right| \leq\left|\mathcal{F}_{j_{2}}\right|$. Similarly, by considering the mapping $\theta_{\mathbf{k}}^{k_{m}-j_{2}+j_{1}}$, we obtain $\left|\mathcal{F}_{j_{2}}\right| \leq\left|\mathcal{F}_{j_{1}}\right|$. So $\left|\mathcal{F}_{j_{1}}\right|=\left|\mathcal{F}_{j_{2}}\right|$. This implies $\left|\mathcal{F}_{1}\right|=\ldots=\left|\mathcal{F}_{k_{m}}\right|$ and hence $|\mathcal{F}|=k_{m}\left|\mathcal{F}_{1}\right|$.

Now let $\mathcal{A}$ be an intersecting sub-family of $\mathcal{F}$. Let $A \in \mathcal{A}$ and $q \in\left[k_{m}-1\right]$. Since $\theta_{\mathbf{k}}^{q}(A) \cap A=\emptyset, \theta_{\mathbf{k}}^{q}(A) \notin \mathcal{A}$. By Proposition $2.2, \theta_{\mathbf{k}}^{q}(A) \in \mathcal{F}$. Thus $\mathcal{A}, \Theta_{\mathbf{k}}^{1}(\mathcal{A}), \ldots, \Theta_{\mathbf{k}}^{k_{m}-1}(\mathcal{A})$ are $k_{m}$ disjoint sub-families of $\mathcal{F}$. So we have $k_{m}|\mathcal{A}| \leq|\mathcal{F}|=k_{m}\left|\mathcal{F}_{1}\right|$. Hence result. 
Remark. Deza and Frankl [4] proved the above result for the special case $\mathcal{F}=\mathcal{P}_{n, n}$. Cameron and $\mathrm{Ku}[3]$ proved that the extremal intersecting sub-families of $\mathcal{P}_{n, n}$ are the stars of $\mathcal{P}_{n, n}$. However, there are translation invariant families $\mathcal{F}$ whose largest intersecting sub-families are not all stars of $\mathcal{F}$. For example, suppose $n \geq 3$ and all the $n$ entries of $\mathbf{k}$ are $n$. Let $\mathcal{E}:=\left\{E \in \mathcal{P}_{n, n}:|E \cap\{(1,1),(2,2),(3,3)\}| \geq 2\right\}$ and take $\mathcal{F}$ to be the translation invariant sub-family of $\mathcal{L}_{\mathbf{k}}$ given by $\mathcal{E} \cup \Theta_{\mathbf{k}}^{1}(\mathcal{E}) \cup \ldots \cup \Theta_{\mathbf{k}}^{n-1}(\mathcal{E})$. Clearly $\mathcal{E}$ is an intersecting sub-family of $\mathcal{F}$ that is not a star of $\mathcal{F}$, and $|\mathcal{E}|=|\mathcal{F}| / n$. By Theorem $2.3, \mathcal{E}$ is a largest intersecting sub-family of $\mathcal{F}$.

Theorem 2.3 enables us to generalize the first part of Theorem 1.5.

Theorem 2.4 Let $\mathcal{F}$ and $m$ be as in Theorem 2.3. Let $\mathcal{A}_{1}, \ldots, \mathcal{A}_{p}$ be cross-intersecting sub-families of $\mathcal{F}$. Then

$$
\sum_{j=1}^{p}\left|\mathcal{A}_{j}\right| \leq \begin{cases}|\mathcal{F}| & \text { if } p \leq k_{m} \\ p \frac{|\mathcal{F}|}{k_{m}}=p|\{F \in \mathcal{F}:(m, 1) \in F\}| & \text { if } p \geq k_{m}\end{cases}
$$

Proof. Similarly to the proof of Theorem 1.5 , we set $k_{n+1}:=p, \mathbf{k}^{\prime}:=\left(k_{1}, \ldots, k_{n}, k_{n+1}\right)$ and $\mathcal{A}:=\bigcup_{j=1}^{p}\left\{A \cup\{(n+1, j)\}: A \in \mathcal{A}_{j}\right\}$, and we have that $\mathcal{A}$ is an intersecting family of size $\sum_{j=1}^{p}\left|\mathcal{A}_{j}\right|$. Let $\mathcal{F}^{\prime}:=\{F \cup\{(n+1, j)\}: F \in \mathcal{F}, j \in[p]\}$. So $\mathcal{A} \subset \mathcal{F}^{\prime} \subseteq \mathcal{L}_{\mathbf{k}^{\prime}}$ and $\left|\mathcal{F}^{\prime}\right|=p|\mathcal{F}|$. Now clearly $\mathcal{F}^{\prime}$ is translation invariant. Thus the result follows from Theorem 2.3 (with $\mathbf{k}^{\prime}$ instead of $\mathbf{k}$ ).

For the particularly interesting case when $\mathcal{F}$ in the above theorem is $\mathcal{P}_{r, s}$ (which has size $\left.\frac{s !}{(s-r) !}\right)$, we suggest the following conjecture about the extremal structures.

Conjecture 2.5 If $\mathcal{A}_{1}, \ldots, \mathcal{A}_{p}$ are cross-intersecting sub-families of $\mathcal{P}_{r, s}$ and $\sum_{j=1}^{p}\left|\mathcal{A}_{j}\right|$ is a maximum, then one of the following holds:

(i) $p<s$ and, for some $q \in[p], \mathcal{A}_{q}=\mathcal{P}_{r, s}$ and $\mathcal{A}_{j}=\emptyset$ for all $j \in[p] \backslash\{q\}$;

(ii) $p>s$ and $\mathcal{A}_{1}=\ldots=\mathcal{A}_{p}=\left\{A \in \mathcal{P}_{r, s}:(a, b) \in A\right\}$ for some $(a, b) \in[r] \times[s]$;

(iii) $p=s$ and $\mathcal{A}_{1}, \ldots, \mathcal{A}_{p}$ are as in (i) or (ii).

If this conjecture is true, then the largest intersecting sub-families of $\mathcal{P}_{r, s}$ are the stars of $\mathcal{P}_{r, s}$. To see this, consider $\mathcal{A}_{1}=\ldots=\mathcal{A}_{p}$ (so the families $\mathcal{A}_{j}$ must be intersecting) and $p>s$ in the above conjecture.

Acknowledgements. The author is indebted to an anonymous referee for careful checking of the paper and suggestions that led to an improvement in the presentation.

\section{References}

[1] C. Berge, Nombres de coloration de l'hypergraphe h-parti complet, in: Hypergraph Seminar (Columbus, Ohio 1972), Lecture Notes in Math., Vol. 411, Springer, Berlin, 1974, 13-20. 
[2] P. Borg, A short proof of a cross-intersection theorem of Hilton, submitted.

[3] P.J. Cameron and C.Y. Ku, Intersecting families of permutations, European J. Combin. 24 (2003) 881-890.

[4] M. Deza and P. Frankl, On the maximum number of permutations with given maximal or minimal distance, J. Combin. Theory Ser. A 22 (1977) 352-360.

[5] M. Deza and P. Frankl, The Erdôs-Ko-Rado theorem - 22 years later, SIAM J. Algebraic Discrete Methods 4 (1983) 419-431.

[6] P. Erdôs, C. Ko and R. Rado, Intersection theorems for systems of finite sets, Quart. J. Math. Oxford (2) 12 (1961) 313-320.

[7] P. Frankl, The shifting technique in extremal set theory, in: C. Whitehead (Ed.), Combinatorial Surveys, Cambridge Univ. Press, London/New York, 1987, pp. 81-110.

[8] A.J.W. Hilton, An intersection theorem for a collection of families of subsets of a finite set, J. London Math. Soc. (2) 15 (1977), 369-376.

[9] A.J.W. Hilton and E.C. Milner, Some intersection theorems for systems of finite sets, Quart. J. Math. Oxford (2) 18 (1967), 369-384.

[10] M.L. Livingston, An ordered version of the Erdós-Ko-Rado Theorem, J. Combin. Theory Ser. A 26 (1979), 162-165. 